\title{
Multi-Platform Mass Media Strategy of Seoul Metropolitan Rapid Transit Corporation (SMRT) : Case Study of SMRT in Korea
}

\author{
Seung-jun Moon*, Tae Woo Kim, Sun Hong Park \\ Dept. of Communication and Information, Inha University, Korea \\ *Corresponding Author: maclean97@inha.ac.kr
}

Copyright $@ 2013$ Horizon Research Publishing All rights reserved

\begin{abstract}
Today, corporate social responsibility (CSR) programs have proliferated among the world's largest corporations. Recently, the role of major corporations in Korea has changed also. Rather than simply making profit, they now have a social role and strive to be accountable to stakeholders. The Seoul Metropolitan Rapid Transit Corporation (SMRT), one of the major public corporations in Korea, spends much money on its multi-platform mass media strategy. The purpose of this paper is to determine whether the Seoul Metropolitan Rapid Transit Corporation (SMRT) is successfully implementing a multi-platform mass media strategy to inform stakeholders of efforts to improve social, economic, and safety environments. A hierarchical regression analysis was used because it is one of the best methods to control for third variables causing pseudo-effects. Finally, it was found out that only newspaper and radio affected respondents' perception of SMRT's social environment. Interestingly, exposure to magazine was negatively related to respondents' perception of SMRT's economic environment. In the case of respondents' perception of SMRT's safety environment, none of the chosen mass media platforms had any affect at all.
\end{abstract}

Keywords Multi-Platform Mass Media Strategy, Media Effects

\section{Introduction}

The Seoul Metropolitan Rapid Transit Corporation (SMRT) in Seoul, Korea is the fourth largest metropolitan subway corporation in the world. Established in 1994, SMRT operates four subway lines, and oversees 200 trains that stop at 148 stations over a distance of $152 \mathrm{KM}$. The first subway system in Korea was begun in 1974, with the Seoul Metropolitan Subway Corporation, now Seoul Metro subway system (Seoul Metro, 2009). However, due to economic development and the subsequent increasing number of commuters, construction of a second set of subway lines began in 1990. Managed by a separate entity called the Seoul Metropolitan Rapid Transit Corporation (SMRT) since 1994, the new subway lines serve 2.7 million passengers per day, and are staffed by 6,000 employees. In addition to essential transportation, SMRT provides social and economic services by promoting culture and tourism; encouraging passengers to explore Korean culture via conveniently accessible subway stations (SMRT, 2010).

Several decades of increasing interest and implementation of corporate social responsibility (CSR) has led to a broader business approach of corporate sustainability management (CSM) (Brown, 2008). Korean corporations are also trying to follow the philosophy of corporate sustainability management (CSM). In keeping with CSM philosophy, SMRT focuses on not only CSR, but on corporate sustainability to address social, economic, and safety environment issues. Focusing on their "Happy Together With Citizens" corporate vision, the SMRT Corporation has been recognized for continuous and consistent excellence in management by the Korean Ministry of Government Administration and Home Affairs. However, although SMRT spends much time and money on corporate social responsibility, citizens still cannot distinguish between the newer SMRT Corporation and the older Seoul Metro subway system. Simultaneously, although many corporations communicate their CSR activities "truthfully," citizens tend to consider such reporting of CSR activities as "greenwashing," or actions designed to mislead stakeholders (Delmas\&Burbano, 2012; Jiang \&Bansal 2003; King \& Lenox, 2000; Perez-Batres, Miller, Pisani, \&Doh, 2012). Thus, SMRT is also concerned about citizens' possibly mislead perceptions of its green campaign. Due to citizens' inability to recognize SMRT's endeavors in social, economic, and safety environments, it is likely that SMRT has failed to inform stakeholders of its activities. Though SMRT uses a multi-platform mass media strategy to inform the public of their campaigns to improve social, economic, and safety environments, stakeholders are still not very 
familiar with their endeavors.

The purpose of this paper is to analyze how SMRT, a major public corporation in Korea, informs stakeholders of its social responsibility via a multi-platform mass media strategy. Although SMRT spends much time and money promoting its social responsibility through four major mass media platforms, not many citizens are aware of the social, economic, or safety benefits of SMRT's efforts toward CSM. Thus, this research attempts to determine which combination of mass media platforms should solve SMRT's problems. The reasons for choosing Korea's SMRT for the case study here is that the CSM philosophy, as well as a multi-platform mass media strategy, is becoming widespread in most Asian countries. The concept of a multi-platform strategy is also popular in European countries. Thus, the outcome of an SMRT case study in Korea is a good way to show the Asian perspective to the rest of the world as well as to discuss strategies that might prove beneficial for European audiences.

\section{Literature Review}

Governments and organizations often use various mass media platforms such as television, newspapers, magazines, radio, and the Internet to manage their social image because massmedia informs stakeholders and focuses their attention on salient issues. The principal aim is to determine the level of visibility of these companies in the general information press, and what the attributes (economic and/or social) are (Capriotti, 2007). SMRT regularly utilizes the four mass media platforms of television, newspaper, magazine, and radio; determined to be the most appropriate mass media platforms for social, economic, and safety campaigns.

Firstly, television is used by many corporations like SMRT as it is a significant mass media platform. Televisionservices not only provide interactive services, but also offer long tail business models with tailored content. Television services can act as a gateway that delivers culture to a wide audience, and television broadcasting is now believed to be a public asset (Evens, De Marez, Hauttekeete, Biltereyst, Mannens, \&Van De Walle, 2010; Lee, 2007, Stadler, 2004).

Secondly, newspaper is another traditional mass media platform, and is even used by governments to try to draw global awareness to events and create positive perceptions (Kim \& Yang, 2008). Trumbo (2012) found that a positive relationship exists in which increases and decreases of newspaper coverage corresponds with civilians' attention on specific issues. Thus, it is believed that the effectof newspaperis more or less confined to attitudes of the public towards specific issues (Bortz, \&Braune, 1980).

Thirdly, magazine should also be another effective mass media platform for SMRT. Compared to other mass media platforms, magazine is less expensive to utilize, with colorful photos as well as detailed articles. Visual images in magazines cause readers to remember the articles for a longer time than articles in newspapers or content on television. In addition, magazines are more flexible and generous with space and time than are television, newspapers or radio; allowing writers to present greater details and information.

Finally, the mass media platform of radio stimulates the creation of mental images in the minds of listeners. Through past research in mental imagery, it has been found that audio cues in radioresult in listeners remembering issues more easily. Specifically, auditory processing of radio with sound effects and sound bites create mental images in the listeners' minds and maintains listeners' attention (Rodero, 2012).

\section{Research Question}

The purpose of this study is to determine which multi-platform mass media strategy should be good for informing stakeholders of SMRT's social, economic, and safety environments. Due to the different characteristics of each mass media platform (e.g. visual and audio cues of television, black and white visual cues of newspaper, full color visual cues of magazines, and audio cues of radio), it is assumed that the effect of content delivery through each mass media platform will be different. Thus, based on the different characteristics of each mass media platform, the following research questions were suggested.

RQ: Which mass media platforms should be relevant for informing stakeholders of SMRT's social, economic, and safety environments? Which combination of mass media platforms should be suggested for SMRT's campaigns?

\section{Sampling}

A survey method was chosen to answer the research questions. Almost 250 questionnaires, which inquired about how much respondents are exposed to SMRT's multi-platform mass media strategy and what respondents think of SMRT's social, economic, and safety environments, were distributed in Seoul, Korea, the main site of SMRT's operation. Any insincere and missing data sets were excluded, with a final count of 218 survey questionnaires analyzed. The demographics of the final data were as follows: Male $=106,48.6 \% ;$ Female $=112,51.4 \%$; Age $=28.83$, S.D. $=9.85$. Respondents were asked to rate their frequency of exposure to information about SMRT on television, newspaper, magazine, and radio using a 7-point semantic differential scale.

In order to find answers to the research questions, the conceptual definitions of both the independent and dependent variables need to be clarified. To evaluate the concept of mass media exposure, two categories of dimensions, the independent variables, were needed: time exposure and content exposure. Time exposure refers to how much the stakeholder is exposed to each medium's coverage of SMRT's campaigns. Content exposure relates to how 
satisfied the stakeholder is with the content of each medium. At the same time, stakeholders' perceptions of SMRT's social, economic, and safety environments, the dependent variables, were evaluated in order to reveal how these mass media strategies affect respondents' perceptions.

\subsection{Time Exposure}

In order to evaluate mass media effects, respondents' time exposure to television, newspaper, magazine, and radio were evaluated using a semantic differential seven-point scale. The range should be from 1 to 7 ; with 1 indicating not at all, and 7 indicating always. In order to evaluate stakeholders' time exposure to television coverage of SMRT, four indicators from the questionnaire were looked at: Never Watch - Always Watch $(\mathrm{M}=2.67)$, Least Detailed - Most Detailed $(\mathrm{M}=2.53)$, Shortest Coverage-Longest Coverage $(\mathrm{M}=2.44)$, and Never Exposed - Always Exposed $(\mathrm{M}=2.65)$. These four indicators evaluating stakeholder time exposure to coverage of SMRT were combined into one dimension of television coverage (Cronbach's alpha $=.93$ ).

To evaluate stakeholders' time exposure to newspaper articles related to SMRT, there were four indicators on the questionnaire: Never Read - Always Read $(\mathrm{M}=2.78)$, Least Detailed - Most Detailed ( $\mathrm{M}=2.81)$, Shortest Coverage Longest Coverage $(\mathrm{M}=2.70)$, Never Exposed - Always Exposed $(\mathrm{M}=2.61)$. The four indicators were then combined into a single dimension of time exposure to newspaper coverage of SMRT (Cronbach's alpha $=.94$ ).

Stakeholders' time exposure to SMRT through magazines was determined by four indicators from the questionnaire: Never Read - Always Read ( $M=2.00)$, Least Detailed - Most Detailed $(M=2.04)$, Shortest Coverage - Longest Coverage $(\mathrm{M}=2.03)$, and Never Exposed - Always Exposed $(\mathrm{M}=1.93)$. One dimension of time exposure to SMRT via newspaper was then calculated by combining the four indicators $($ Cronbach's alpha $=.92)$.

Finally, four indicators were used to evaluate stakeholders' time exposure to SMRT through radio: Never Heard Always Heard $(\mathrm{M}=2.28)$, Least Detailed - Most Detailed $(\mathrm{M}=2.23)$, Shortest Coverage - Longest Coverage $(\mathrm{M}=2.14)$, and Never Exposed - Always Exposed $(\mathrm{M}=2.18)$. The final four indicators were combined into a dimension to determine stakeholders' time exposure to SMRT campaigns via the media platform of radio (Cronbach's alpha $=.94$ ).

\subsection{Content Exposure}

Content exposure means how much the stakeholder should be satisfied with the content of each medium, i.e. television, newspaper, magazine, and radio. In order to evaluate the content of SMRT through television exposure, four indicators of content were evaluated using the seven-point scale: Worst Quality - Excellent Quality $(\mathrm{M}=3.12)$, Least Recent - Most Recent $(\mathrm{M}=3.07)$, Least Helpful - Most Helpful ( $M=3.03)$, and Worst Impression Best Impression $(\mathrm{M}=2.91)$. These four indicators were combined to create an index of respondents' content exposure to SMRT campaigns through television $($ Cronbach's alpha $=.94)$.

Four indicators were then evaluated for newspaper content covering SMRT's campaigns: Worst Quality - Excellent Quality ( $\mathrm{M}=3.0154)$, Least Recent - Most Recent $(\mathrm{M}=2.81)$, Least Helpful - Most Helpful $(\mathrm{M}=2.93)$, and Worst Impression - Best Impression ( $\mathrm{M}=2.87)$. All four indicators were combined to form a content exposure index of SMRT campaigns from newspaper (Cronbach's alpha $=.93$ ).

Content of SMRT campaigns from another print mass media platform, magazine, was evaluated using the four indicators for content: Worst Quality - Excellent Quality $(\mathrm{M}=2.53)$, Least Recent - Most Recent $(\mathrm{M}=2.45)$, Least Helpful - Most Helpful $(\mathrm{M}=2.46)$, and Worst Impression Best Impression $(\mathrm{M}=2.44)$. The next step combined these four indicators of magazine content into one index of respondents' content exposure (Cronbach's alpha $=.93$ ).

Finally, SMRT content presented by the audio mass media platform of radio was evaluated using four indicators: Worst Quality - Excellent Quality ( $\mathrm{M}=2.61)$, Least Recent - Most Recent ( $\mathrm{M}=2.59)$, Least Helpful - Most Helpful ( $\mathrm{M}=2.53)$, and Worst Impression - Best Impression $(\mathrm{M}=2.38)$. An index of respondents' content exposure to SMRT campaigns via radio was created by combing all four indicators (Cronbach's alpha $=.92$ ).

Finally, in order to measure respondents' SMRT mass media exposure, a factor analysis was performed for the dimensions of time exposure and content exposure. The results of the factor analysis showed two clear dimensions, time exposure and content exposure. Thus, all indicators of each dimension in each medium were combined to measure time exposure and content exposure of each medium. Finally the exposure of each mass media was evaluated based on the combination of the two dimensions.

\subsection{Stakeholders' Perception on SMRT's Environments}

SMRT's environments consist of three dimensions, thus, respondents were asked their perception of SMRT's social, economic, and safety environments. Firstly, the conceptual definition of the dimension "social environment" consists of stations with nice views promoted as part of SMRT Culture $\&$ Tourism, a culture gallery at each station, and focus on the "Happy Together With Citizens" corporate vision. In order to evaluate the social environment, four indicators from the questionnaire were analyzed: Least Variety of Services Most Variety of Services ( $\mathrm{M}=3.61)$, Worst Customer Service - Best Customer Service (M=3.57), Least Cultural Services - Most Cultural Services ( $M=3.52)$, and Least Philanthropic - Most Philanthropic $(\mathrm{M}=2.86)$. The indicators were then combined into one index for respondents' perception of SMRT's social environment (Cronbach's alpha $=.82$ ).

Secondly, the conceptual definition of "economic environment" is the degree to which SMRT decreases traffic congestion and pollution, and provides an affordable transportation alternative that is ecologically friendly. Three 
indicators on the questionnaire were evaluated for economic environment: Worst Transfer Facilities - Best Transfer Facilities $(\mathrm{M}=5.18)$, Least Transportation Savings - Most Transportation Savings $(\mathrm{M}=5.00)$, and Never Reduces Congestion - Always Reduces Congestion ( $\mathrm{M}=5.38)$. These three indicators were combined into a single dimension for respondents' perception of SMRT's economic environment (Cronbach's alpha $=.81$ ).

Thirdly, "safety environment" focuses on frequency of accidents, secure stations, and punctual schedule at safe speeds. Four indicators for "safety environment" were evaluated: Worst Safety Record - Best Safety Record $(\mathrm{M}=4.04)$, Least Trustworthy - Most Trustworthy $(M=4.07)$, Worst Travel Safety - Best Travel Safety $(\mathrm{M}=4.68)$, and Worst Customer Service - Best Customer Service ( $M=3.69)$. To form one dimension of respondents' perception of SMRT's safety environment, these four indicators were then combined (Cronbach's alpha $=.83$ ).

A factor analysis was performed with all indicators, and showed that designated indicators should be grouped based on each dimension of social, economic, and safety environment. Thus, it was assumed that each indicator represents each dimension.

\section{Results}

This study focused mainly on how SMRT's current multi-platform mass media strategy of using television, newspaper, magazine, and radio affects stakeholders' perceptions of SMRT's social, economic, and safety environments. However, variables other than mass media exposure might have already affected stakeholders' perceptions of these three dimensions, resulting in pseudo-effects. In order to prevent pseudo-effects, hierarchical regression analysis was used because it is one of the best methods to control for variables causing pseudo-effects (see Table 1).

In particular, the effects of SMRT's mass media strategy were tested to see whether or not they affected stakeholders' perceptions of SMRT's social, economic, and safety environments. However, aside from SMRT's mass media strategies, respondents' gender, age, their existing image of SMRT, and the characteristics of SMRT may affect the dependent variables - that is, respondents' perceptions of SMRT's social, economic, and safety environments. The effects of all potential variables like gender, age, corporate image, and corporate characteristics were controlled for using hierarchical regression analysis.

Table 1. Hierarchal regression model

\begin{tabular}{|c|c|c|c|}
\hline & $\begin{array}{l}\text { Social environment } \\
\text { Final B }\end{array}$ & $\begin{array}{l}\text { Economic environment } \\
\text { Final B }\end{array}$ & $\begin{array}{l}\text { Safety environment } \\
\text { Final B }\end{array}$ \\
\hline \multicolumn{4}{|l|}{ Demographics } \\
\hline Gender & -.08 & -.07 & -.08 \\
\hline Age & $.12^{*}$ & -.03 & .11 \\
\hline Usage experience & .03 & $.11^{*}$ & $.17^{* *}$ \\
\hline $\mathbf{R}^{2}$ & $3.6 \% *$ & $4.6 \% *$ & $4.4 \% *$ \\
\hline \multicolumn{4}{|c|}{ Corporate Information } \\
\hline Image & $.5^{* * *}$ & $.48^{* * *}$ & $.42 * * *$ \\
\hline Characteristics & $.14^{*}$ & $.29 * * *$ & $.24 * * *$ \\
\hline $\mathbf{R}^{2}$ & $40.8 \% * *$ & $39.2 \% * * *$ & $34.5^{* * *}$ \\
\hline \multicolumn{4}{|l|}{ Mass media } \\
\hline Television & -.06 & -.06 & -.09 \\
\hline Newspaper & $.17^{*}$ & .11 & .07 \\
\hline Magazine & -.01 & $-.16^{*}$ & .01 \\
\hline Radio & $.21^{*}$ & -.04 & .1 \\
\hline $\mathbf{R}^{2}$ & $8.1 \% * *$ & $2.5 \% *$ & $1.1 \%$ \\
\hline
\end{tabular}

${ }^{*} \mathrm{p}<.05, * * \mathrm{p}<.01, * * * \mathrm{p}<.001 ;$ all entries are final standardized coefficients $(\mathrm{n}=218)$ 
First, in order to test how mass media exposure affects respondents' perception of SMRT's social environment, all potential variables, including mass media exposure, should be analyzed. Thus, effects of demographics variables which may affect the three environment dimensions were controlled. It was found that demographic variables such as gender $(\beta=-.08 ; p>.05)$ and usage experience $(\beta=.03 ; p>.05)$ did not affect the perception of SMRT's social environment (see Table 1). However, the variable of age is a positive predictor for social environment $(\beta=.12 ; \mathrm{p}<.05)$. This means that the older one is, the more one thinks positively about social environment. This first block of demographics $\left(\mathrm{R}^{2}=3.6 \% ; \mathrm{p}<.05\right)$ accounted for a significant portion of the variance. After controlling for the demographic block, the second block of corporate information was analyzed. Both corporate image $(\beta=.50 ; \mathrm{p}<.001)$ and characteristics $(\beta=.14$; $\mathrm{p}<.01)$ were significant predictors for respondents' perception of SMRT's social environment. Lastly, after controlling for both the demographic block and the corporate information block, the mass media block was analyzed. Although television $(\beta=-.06 ; p>.05)$ and magazine $(\beta=-.01 ; p>.05)$ were not related to perception of SMRT's social environment, newspaper $(\beta=.17 ; p<.05)$ and radio $(\beta=.21 ; p<.05)$ were positively related. Only newspaper and radio affected social environment, and the third block of mass media $\left(\mathrm{R}^{2}=8.1 \% ; \mathrm{p}<.05\right)$ accounted for a significant portion of the variance.

Second, in order to test how mass media exposure affects respondents' perception of SMRT's economic environment, variables which may affect perception of economic environment were controlled. It was found that gender $(p>.05, \beta=-.07)$ and age $(p>.05, \beta=-.03)$ were not related to perception of SMRT's economic environment while the variable of usage experience $(\beta=.11 ; \mathrm{p}<.05)$ was statistically significant. The first block, demographics $\left(\mathrm{R}^{2}=4.6 \% ; \mathrm{p}<.05\right)$, accounted for a significant portion of the variance. In the second block, corporate information, the variables of image $(p<.001, \beta=.48)$ and characteristics $(p<.001, \beta=.29)$ were related to economic environment. After controlling for both the first and second blocks, the mass media block was analyzed. In particular, magazine $(\mathrm{p}<.05, \beta=-.16)$ was negatively related to respondents' perception of SMRT's economic environment, while television ( $p>.05, \beta=-.06)$, newspaper $(p>.05, \beta=.11)$, and radio $(p>.05, \beta=-.04)$ were not related, either positively or negatively, to perception of economic environment. This means that the more people read magazines covering the SMRT Corporation, the less positive they feel about its economic environment.

Third, the effects of potential variables on respondents' perception of SMRT's safety environment were analyzed. Neither gender $(p>.05, \beta=-.08)$ nor age $(p>.05, \beta=.11)$ was a significant predictor of perception of safety environment. However, usage experience ( $p>01, \beta=.17)$ was statistically significant for perception of safety environment. After controlling for the demographic block, the second block of corporate information was analyzed. The images $(\mathrm{p}<.001$, $\beta=.42)$ and characteristics $(p<.001, \beta=.24)$ of SMRT were significant for safety environment.

After controlling for the corporate information block, the third block of mass media exposure was analyzed. However, all the mass media platforms of television $(p>.05, \beta=-.09)$, newspaper $(p>.05, \beta=.07)$, magazine $(p>.05, \beta=.01)$ and radio $(p>.05, \beta=.1)$ were not predictors of perception of SMRT's safety environment. This regression model predicting safety environment accounted for a total of $1.1 \%$ of the variance.

\section{Conclusion}

The purpose of this paper is to see how SMRT, a major public corporation in Korea, informs stakeholders of its social responsibility via multi-platform mass media strategy. Currently, although SMRT uses a multi-platform mass media strategy of television, newspaper, magazine and radio, stakeholders are not aware of the corporation's efforts to improve social, economic, and safety environments. This study used a hierarchal regression to diagnose how SMRT's current multi-platform mass media strategy affects citizens' perception of SMRT's campaigns.

First, with the exception of the variable of age, the first block, gender and usage experience were not statistically significant for respondents' perception of SMRT's social environment. Also, after excluding the effects of all variables of the demographics and corporation information blocks, only newspaper and radio were found to be significant mass media platforms for informing stakeholders of SMRT's social environment. Interestingly, the mass media platform of magazine was found to be negatively related to respondents' perception of economic environment. This is somewhat of a serious problem, and the content informing stakeholders of SMRT's economic environment in magazines should be changed to address this finding. Also, it was revealed that the mass media platforms of television, newspaper, and radio were not related to perception of SMRT's economic environment at all. Even though SMRT spends much of its budget to inform stakeholders of its economic environment, the chosen mass media platforms of television, newspaper, and radio were not influential on respondents' perception. Moreover, magazine was negatively significant for perception of SMRT's economic environment. Finally, no mass media platform affected respondents' perception of SMRT's safety environment. Thus, a new multi-platform mass media strategy for increasing positive images of economic and safety environments should be considered as well.

Second, although major companies in Korea often use many mass media platforms, they have not empirically recognized which mass media platforms are significant, or how much each mass media platform affects company goals. This study empirically showed which mass media platforms affected, or did not affect, stakeholders, and clearly showed the process of analyzing mass media effects. Thus, the three dimensions of social, economic, and safety environment 
were empirically analyzed based on the strategic purpose of each mass media platform. For example, mass media platform affects $8.1 \%$ of respondents' perception of SMRT's social environment, and $2.5 \%$ of respondents' perception of SMRT's economic environment. In the case of SMRT's safety environment, a multi-platform mass media strategy did not have any affect at all. The results of this research are different from previous strategies of most corporations that simply allocated part of the corporate budget and used intuition to manage each multi-platform mass media platform.

Finally, this study is special in that the effects of all potential variables were controlled with a hierarchal regression. As seen in Table 1, the second block of corporate information accounted for a large portion of social environment (40.8\%), economic environment (39.2\%), and safety environment (34.5\%). This is an expected outcome because the subway system especially that of SMRT, has been used by Koreans for several decades as an integral part of life in Seoul, Korea, and corporate information about SMRT has been widely spread among Koreans. Thus, the effects of corporate information are much larger than any other variables, including multi-platform mass media platforms. In this study, after screening out the large influence of corporate information, all mass media effects were analyzed in order to remove pseudo effects. It means that this study successfully analyzed the empirical relationship between independent variables and dependent variables without pseudo effects, and with high internal validity; specifically relations between SMRT's multi-platform mass media strategy and stakeholders' perception of SMRT's social, economic, and safety environments.

\section{REFERENCES}

[1] Bortz, J. \&Braune, P. (1980). The effects of daily newspapers on their readers--Exemplary presentation of a study and its results. European Journal of Social Psychology, 10(2), 165-193.

[2] Brown, R. E. (2008). Sea change: Santa Barbara and the eruption of corporate social responsibility. Public Relations Review, 34(1), 1-8.

[3] Capriotti, P. (2007). The corporate social responsibility of Spanish companies in the mass media. Revista de Estudios de Comunicacion, 12(23), 61-74.

[4] Delmas, M. A., \&CuerelBurbano, V. (2011). Environmental management and regulatory uncertainty. California Management Review-SI, 54(1), 64-87.

[5] Evens, T., De Marez, L., Hauttekeete, L., Biltereyst, D. Mannens, E., \& Van De Walle, R.(2010). Attracting the un-served audience: the sustainability of long tail-based business models for cultural television content. New Media \& Society, 12(6), 1005-1023.
[6] Jiang, R. H. J., \&Bansal, P. (2003). Seeing the need for ISO 14001. Journal of Management Studies, 40(4), 1047-1067.

[7] Kim, J., \& Yang, S. (2008). Effects of government public relations on international news coverage. Public Relations Review, 34(1), 51-53.

[8] King, A. \& Lenox, J. (2000). Industry self-regulation without sanctions: The chemical industry's responsible care program. Academy of Management Journal, 43(4), 698-716.

[9] Lee, S. (2007). International public relations as a predictor of prominence of US news coverage. Public Relations Review, 33(2), 158-165.

[10] Perez-Batres, L., Miller, V., Pisani, M., \&Doh, J. (2012). Stakeholder pressures as determinants of CSR strategic choice: why do firms choose symbolic versus substantive self-regulatory codes of conduct? Journal of Business Ethics, 110:157-172.

[11] Rodero, E. (2012). See it on a radio story: sound effects and shots to evoked imagery and attention on audio fiction. Communication Research, 39(4), 458-479.

[12] Roscoe, J. (2004). Multi-platform event television: Reconceptualizing our relationship with television. Communication Review, 7(4), 363-369.

[13] Seoul Metro. (2009). Retrieved from http://www.seoulmetro.co.kr/eng/.

[14] SMRT. (2010). Retrieved from http://www.smrt.co.kr/main/index/index002.jsp.

[15] Stadler, J. (2004). AIDS ads: make a commercial, make a difference? Corporate Social Responsibility and the Media. Journal of Media and cultural studies, 18(4), 591-610.

[16] Trumbo, C. (2012). The effect of newspaper coverage of influenza on the rate of physician visits for influenza 2002-2008. Mass Communication \& Society, 15(5), 718-738. 